\title{
Cetuximab verbessert die Wirksamkeit der Chemotherapie in allen Therapielinien
}

\begin{abstract}
Der epidermale Wachstumsfaktor (EGF) wirkt über einen spezifischen Rezeptor (EGFR) stimulierend auf Tumorzellen. $\mathrm{Zu}$ den Zellen, die in starkem Ausmaß EGFR exprimieren, gehören das kolorektale Karzinom und Plattenepithelkarzinome des Kopf-Hals-Bereichs. Beim fortgeschrittenen kolorektalen Karzinom hat der monoklonale Anti-EGFR-Antikörper Cetuximab $\left(\right.$ Erbitux $\left.^{\circledR}\right)$ in den letzten Jahren in zahlreichen Phase-II- und Phase-III-Studien gezeigt, dass er in allen Stadien der Therapie das progressionsfreie Überleben, die Ansprechrate und teilweise auch das Gesamtüberleben verlängert.

Zugelassen ist Cetuximab bislang in Kombination mit Irinotecan zur Behandlung von Patienten mit EGFR-exprimierenden metastasierten kolorektalen Karzinomen nach Irinotecan-Versagen. In Kombination mit Standard-Chemotherapien stellt der Antikörper aber auch für nicht vorbehandelte Patienten mit metastasiertem kolorektalem Karzinom (mCRC) eine wirksame Therapieoption dar. Dies zeigt insbesondere die CRYSTAL-Studie, deren Ergebnisse ClausHenning Köhne, Oldenburg, auf der Jahrestagung der Deutschen, Österreichischen und Schweizerischen Gesellschaften für Hämatologie und Onkologie (DGHO) präsentierte:

gruppe von Patienten zu sein, deren Metastasen sich ausschließlich auf die Leber beschränkt hatten: In dieser Subgruppe ist der progressionsfreie Überlebensvorteil nach Cetuximab-Therapie mit $11,4$ versus 9,2 Monaten ( $p=0,023)$ noch stärker ausgeprägt als im Gesamtkollektiv. Die zusätzliche Gabe von Cetuximab konnte bei diesen Patienten das Risiko der Tumorprogression um $36 \%$ reduzieren.

-Weiterhin wurde auch der sekundäre Endpunkt der Studie, die signifikante Erhöhung der Ansprechrate, erreicht: In der Cetuximab-Gruppe wurde eine Tumorverkleinerung in $47 \%$, im Kontrollarm in nur 39\% der Fälle beobachtet ( $\mathrm{p}$ $=0,005$, Abb. 1 links).

- Alle Patienten waren ursprünglich als nicht operabel betrachtet worden. Nach der Therapie war der Anteil an Patienten, in denen eine R0Resektion erreicht werden konnte, in der Cetuximab-Gruppe nahezu dreimal so hoch $(\mathrm{p}=$ 0,0034, Abb. 1, Mitte) wie in der Gruppe mit FOLFIRI alleine.

- Die für Cetuximab bekannten Hautreaktionen traten auch in dieser Studie auf, und interessanterweise korrelierte ihr Ausmaß, wie das auch schon in anderen Studien gezeigt wurde, mit dem progressionsfreien Überleben.
\end{abstract}

Die CRYSTAL-Studie (Cetuximab combined with iRinotecan in first-line therapY for metaSTatic colorectAL cancer) ist eine randomisierte, kontrollierte, internationale Phase-III-Studie, in der die Standard-Kombinationschemotherapie FOLFIRI (5-Fluorouracil, Folinsäure, Irinotecan) plus Cetuximab bei nicht vorbehandelten Patienten mit mCRC mit einer alleinigen FOLFIRI-Therapie verglichen wurde. Mit über 1.200 eingeschlossenen Patienten ist CRYSTAL eine der bislang größten klinischen Untersuchungen zur Wirksamkeit von Cetuximab.

- Beim primären Endpunkt der Studie, dem progressionsfreien Überleben (PFS) wurde im Cetuximab-Arm eine statistisch signifikante Verlängerung $(8,9 \mathrm{im}$ Vergleich $\mathrm{zu}$ 8,0 Monate im FOLFIRI-Arm, $\mathrm{p}=0,048$ ). Die 1-Jahres-PFS-Rate lag im CetuximabArm bei $34 \mathrm{im}$ Vergleich zu 23\% unter FOLFIRI allein. Insgesamt konnte der Einsatz von Cetuximab das Risiko einer Tumorprogression um $15 \%$ senken.

- Noch günstiger schien die Situation einer exploratorischen Analyse zufolge in der Subtuximab $(\mathrm{n}=122)$.
Diese ermutigenden Ergebnisse, so Köhne, belegen nicht nur, dass mit Cetuximab eine neue Option für die Erstlinienbehandlung des metastasierten kolorektalen Karzinoms zur Verfügung steht. Sie zeigen darüber hinaus die konsistent gute Wirksamkeit von Cetuximab in allen Therapielinien. Das alles ist gegenüber der reinen Chemotherapie mit einer kaum erhöhten Nebenwirkungsrate behaftet: Neben den für AntiEGFR-Therapien charakteristischen Hautreaktionen war lediglich der Anteil an Diarrhöen etwas erhöht (15,2 vs. 10,5\%).

OPUS: Auch in Kombination mit Oxaliplatin-haltigen Regimen hat Cetuximab in Phase-II-Studien viel versprechende Wirksamkeit in der First-line gezeigt. Deshalb, so Carsten Bokemeyer, Hamburg, wurden in der europaweiten OPUS-Studie (OxaliPlatin and cetUximab in firSt-line treatment of mCRC) Patienten mit fortgeschrittenem kolorektalem Karzinom in zwei Gruppen randomisiert: FOLFOX4 mit oder ohne Cetuximab in der üblichen Dosierung von initial $400 \mathrm{mg} / \mathrm{m}^{2}$ und anschließend wöchentlich $250 \mathrm{mg} / \mathrm{m}^{2}$. Beim primären Endpunkt, der Ansprechrate, war die Chemoimmuntherapie mit 45,6 vs. $35,7 \%$ überlegen.

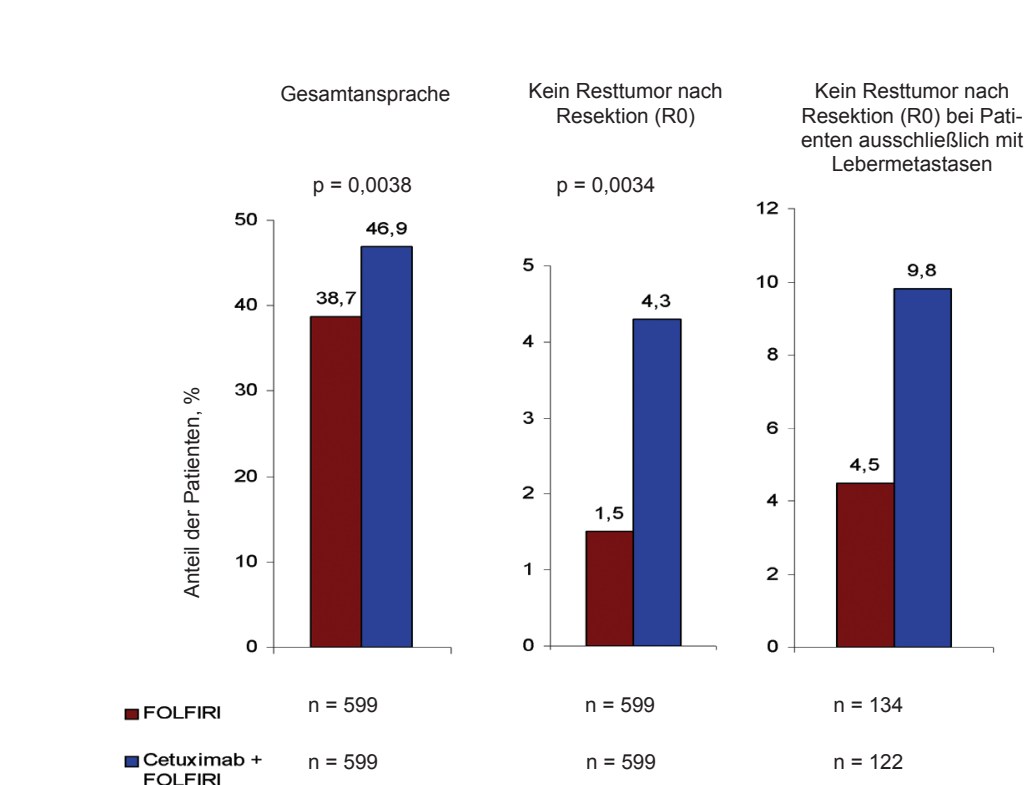

Abb. 1. CRYSTAL-Studie: Ansprechraten aller Patienten (links) sowie R0-Resektionsraten aller Patienten (Mitte) und der Patienten, die lediglich Lebermetastasen aufwiesen (rechts) unter FOLFIRI $(n=134)$ bzw. FOLFIRI plus Ce- 


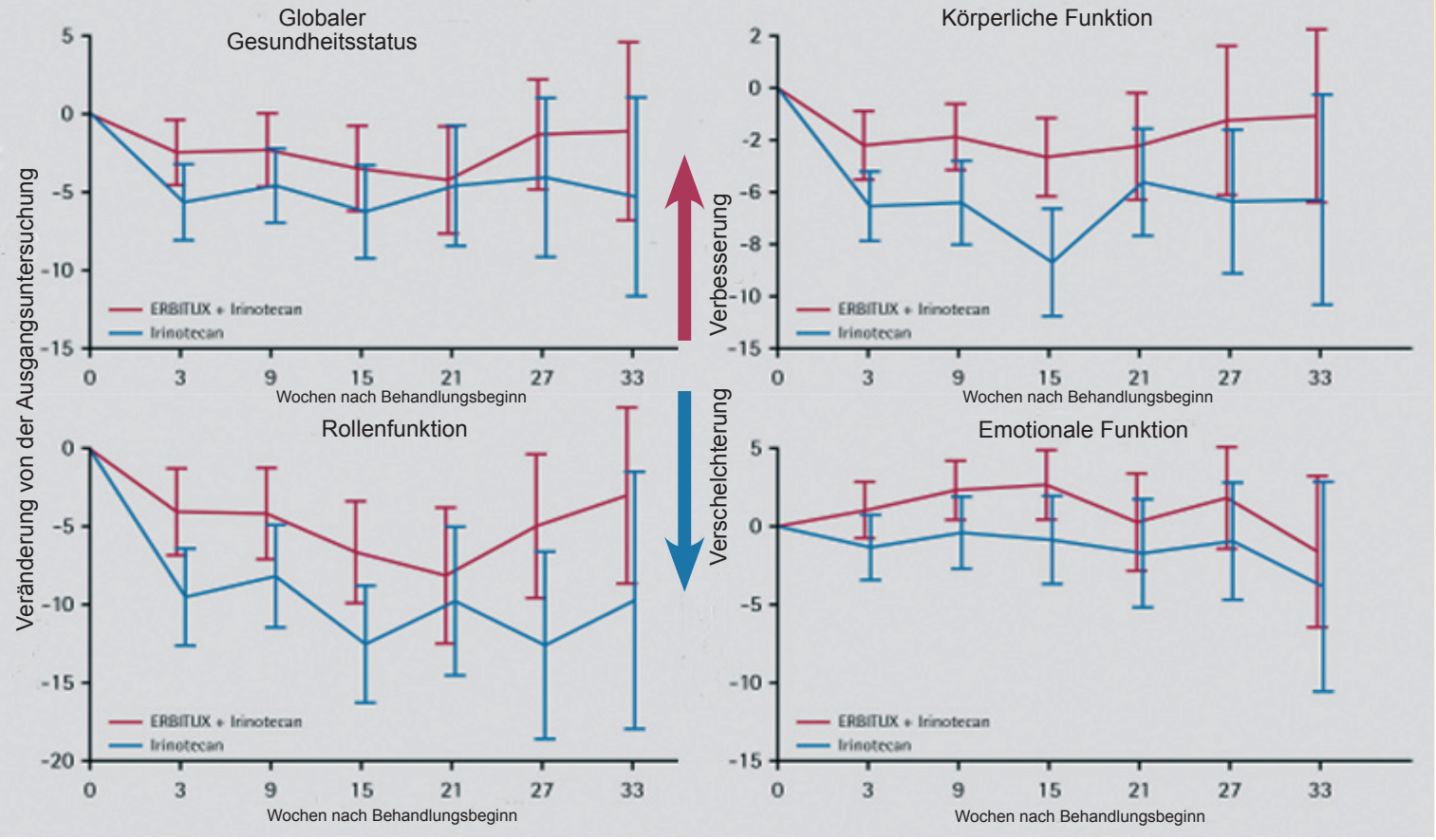

Abb. 2. EPICStudie: Veränderung verschiedener Lebensqualitäts-Parameter (gelb: Cetuximab plus FOLFIRI, grün: FOLFIRI alleine).

\section{EPIC: Verbesserung von Ansprechen, PFS und Lebensqualität in der Second-line}

Neben den First-line-Daten gibt es auch neue Ergebnisse zum Nutzen von Cetuximab bei der Therapie vorbehandelter Patienten. Die PhaseIII-Studie EPIC (European Prospective Investigation of Cancer) untersuchte Cetuximab plus Irinotecan versus Irinotecan alleine bei 1.300 Patienten mit mCRC, die auf eine vorangegangene FOLFOX-Chemotherapie nicht mehr angesprochen hatten, die also nicht die derzeitigen Zulassungskriterien (Irinotecan-Versagen) erfüllten. Die Daten zeigten nach den Worten von Gunnar Folprecht, Dresden, eine Vervierfachung des Ansprechens (16 vs. 4\%) und ein längeres progressionsfreies Überleben unter Cetuximab (4,0 vs. 2,6 Monate). Das Gesamtüberleben war mit 10,7 vs. 10,0 Monaten nicht signifikant verlängert, was mit großer Wahrscheinlichkeit darauf zurückzuführen ist, dass etwa die Hälfte der Patienten in der Kontrollgruppe nach Abschluss der Studie ebenfalls Cetuximab bekam.

Dagegen zeigte eine ausführliche Analyse, dass neben dem progressionsfreien Überleben und der Ansprechrate auch die gesundheitsbezogene Lebensqualität im Cetuximab-Arm signifikant im Vergleich zum Irinotecan-Arm alleine verbessert wird. Der Erhebungsbogen der European Organization for Research and Treatment of Cancer (EORTC) zur Bestimmung der Lebensqualität (QLQ-EORTC C30), wurde von der Mehrzahl der Patienten ausgefüllt und zeigte einen signifikanten positiven Effekt im Cetuximab-Arm in 10 von 15 Bereichen. Dazu zählten neben dem globalen Gesundheitsstatus ( $\mathrm{p}=$ $0,049)$ sowohl funktionelle Scores wie körperliche, Rollen- und emotionale Funktionen (Abb. 2) als auch einzelne Symptome, darunter Fatigue, Nausea $(\mathrm{p}<0,0001)$ und Erbrechen, Schmerzen $(p<0,0001)$ und Diarrhö.

EPIC ist damit die erste Studie, so Folprecht, in der bei Zugabe eines «biologischen» Moleküls zu einer Zytostatika-Therapie die Lebensqualität länger erhalten blieb. Er bezeichnete es als sehr ermutigend, dass der signifikante klinische Vorteil von Cetuximab auch subjektiv für den Patienten spürbar wird.

\section{Cetuximab verbessert Überleben und Lebensqualität auch bei stark vorbe- handelten Patienten}

Die Lebensqualität wird durch Cetuximab-Gabe sogar bei stark vorbehandelten Patienten verbessert: In der kanadischen NCIC CTG CO.17-Studie wurden 572 Patienten mit fortgeschrittenem kolorektalem Karziniom, die für alle empfohlenen Therapien refraktär oder intolerant waren, auf eine Cetuximab-Monotherapie oder «Best Supportive Care» (BSC) randomisiert. Neben dem primären Endpunkt, Verlängerung des Gesamtüberlebens, wurde auch die körperlichen Funktion und der globalen Gesundheits-Scores nach 8 und 16 Wochen untersucht.

Cetuximab erhöhte nicht nur, wie Folprecht zeigte, Ansprechrate, progressionsfreies und als erste «biologische» Monotherapie auch das Ge- samtüberleben, sondern der Antikörper brachte auch eine signifikante verbesserte gesundheitsbezogene Lebensqualität als BSC alleine: Patienten unter Cetuximab erfuhren signifikant seltener eine Verschlechterung der gesundheitsbezogenen Lebensqualität und wenn, dann trat sie später auf. Die signifikante Verbesserung erstreckte sich auf alle gemessenen Symptome wie Schmerzen, Fatigue, Nausea, Dyspnoe und sogar die finanzielle Situation.

Cetuximab hat damit gezeigt, dass es in allen Behandlungslinien des fortgeschrittenen kolorektalen Karzinoms eine Verlängerung des progressionsfreien Überlebens und eine Erhöhung der Ansprechrate bei verbesserter Lebensqualität bewirkt.

Josef Gulden, Grafrath

\section{Quelle}

Satellitensymposium «Neue Phase-III-Daten beim mCRC: Erbitux ${ }^{\circledR}$ - der Schlüssel zu mehr Wirksamkeit» während der Jahrestagung der Deutschen Gesellschaft für Hämatologie und Onkologie in Basel, 5. Oktober 2007.

\section{Impressum}

First-line und vorbehandelte Patienten beim kolorektalen Karzinom

Cetuximab verbessert die Wirksamkeit der Chemotherapie in allen Therapielinien PharmaForum in ONKOLOGIE 30 | 12 | 07

(C) 2007 by S. Karger Verlag, 79115 Freiburg, Deutschland

Mit freundlicher Unterstützung durch die Merck Pharma GmbH.

\section{KARGER ○ 2007 S. Karger GmbH, Freiburg}

